Background Pneumonia is a leading cause of hospital admission. With mortality exceeding 18\% (BTS CAP Audit 2013) the search for strategies to reduce this continues. Statins are receiving increasing attention for a potential role in improving survival from acute bacterial infections. MHRA guidance recommends that statins should be paused during treatment with a macrolide due to risk of myopathy and rhabdomyolysis.

We undertook a retrospective study to determine the frequency of concurrent statin and macrolide administration in patients diagnosed with pneumonia and whether concurrent use of a statin and macrolide antibiotic to treat pneumonia improved survival compared to stopping statin treatment, and whether concurrent use was safe and tolerable.

165 patient episodes were identified by searching for patients who were coded as having pneumonia and were prescribed a statin and macrolide. Data was collected on statin, dose, severity of pneumonia including intensive care admission, comorbidities, survival, renal and liver function.

Results $62 \%$ of the cohort continued a statin throughout pneumonia treatment. In the continued statin group survival to hospital discharge was $79 \%$ versus $64 \%$ in the group in whom the statin was paused $(\mathrm{p}=0.034)$.

Severity of pneumonia (CURB score) was similar for both groups. Statin users were less likely to be admitted to intensive care $(28 \%$ vs $46 \%, \mathrm{p}=0.0219)$. Charlson comorbidity index score was similar for the statin $(6.4, \mathrm{IQR}=5-8)$ and non-statin (6.1, IQR $=5-8)$ groups.

There was no increased risk of acute liver, kidney injury or myopathy in the continued statin group.
Conclusion Continued statin use during treatment for pneumonia with a macrolide antibiotic is safe and may improve survival compared to stopping statin use. Current guidance on concurrent use of statins and macrolides should be reviewed.

\section{P266 OUTCOMES FROM THE INTRODUCTION OF FUNGAL BIOMARKERS TO THE NEUTROPENIC FEVER PATHWAY IN A TERTIARY HAEMATOLOGY DEPARTMENT}

R Swayne, D Enoch, S Aliyu, C Crawley, P Krishnamurthy, J Craig, G Follows, B Uttenthal," J Babar, CR Sander. Cambridge University NHS Foundation Trust, Cambridge, UK

\subsection{6/thoraxjnl-2016-209333.409}

Background Invasive fungal disease (IFD) frequently occurs in febrile neutropenic haemato-oncology patients (NHP). Until 2012 no biomarkers were available for the diagnosis of IFD in our Trust. The neutropenic fever pathway was modified to include serial serum Aspergillus galactomannan (GM), serum Aspergillus PCR (APCR) from national reference laboratory and Bronchoalveolar lavage (BAL) GM and APCR.

We compared 299 NHP who were investigated with the original pathway between October 2009 and April 2012 with 307 NHP investigated with the novel pathway between April 2013 and 2015. Primary end point was non-inferiority of novel pathway in terms of 12 month mortality. Secondary outcomes were 30 day mortality, length of treatment, length of stay and confidence of diagnosis based on EORTC/MSG criteria ${ }^{1}$ and concordance of the different biomarkers.

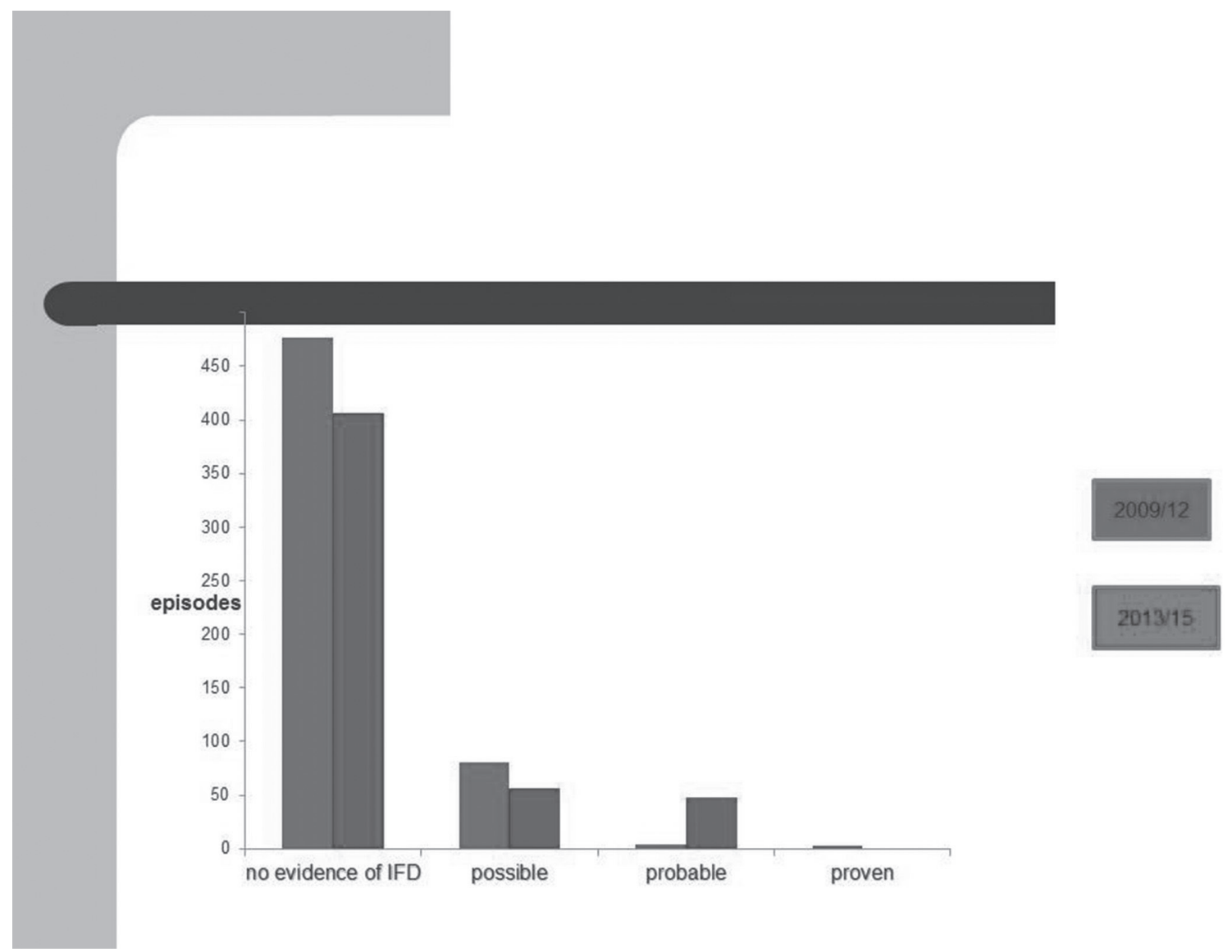

Abstract P266 Figure 1 Increased confidence in diagnosis of IFD according to EORTC/MSG criteria with novel pathway 
Method Prospective patients (2013-15 cohort) were identified from ward lists. Retrospective patients (2009-12 cohort) were identified from haemato-oncology patients who had had blood cultures and were co-incidentally found to be neutropenic. Medical notes, drug charts, discharge letters and microbiology results were reviewed.

Results The 2 cohorts were well matched in terms of haematooncology diagnosis. The 2009/12 cohort included 561 episodes, with 333 CT chests and 62 bronchospies compared to 508 episodes, 288 CT chests and 86 bronchoscopies in 2013/15 group. 12 month mortality was $42 \%$ in $2009 / 12$ versus $34 \%$ in $2013 / 15$ cohort. 30 day mortality was $11 \%$ for both cohorts. There was no significant difference in length of antifungal treatments, although $24 \%$ switched to voriconazole following positive biomarkers.

Concordance between serum and BAL GM was $14.8 \%$ and APCR was 6.3\%. Concordance between serum GM and APCR was $7 \%$ and between BAL GM and PCR was $41 \%$.

Confidence in diagnosis of IFD increased with the novel pathway See Figure 1.

Conclusion The introduction of a novel NF pathway was found to be non-inferior in terms of 12 month and 30 day mortality. Although there was increased confidence in the diagnosis of IFD, this did not translate to reduced antifungal treatment, although it did influence switching to voriconazole and secondary chemoprophylaxis. Negative serum GM and PCR did not rule out the diagnosis of IFD and BAL biomarkers were more sensitive than serum ones.

\section{REFERENCE}

1 De Pauw B, et al. Clin Infect Dise 2008;46(12).

\section{P267 DLCO PREDICTS DISEASE SEVERITY AND MORTALITY IN BRONCHIECTASIS}

MJ McDonnell, M O'Mahony, D Breen, JJ Gilmartin, A O'Regan, RM Rutherford. Galway University Hospitals, Galway, Ireland

\subsection{6/thoraxjnl-2016-209333.410}

Background Few studies have assessed the role of lung diffusing capacity (DLCO\%) in bronchiectasis. We sought to examine the relationship between DLCO\% and clinical and radiological variables in a well-defined population of bronchiectasis patients to determine its potential prognostic significance and compare with $\mathrm{FEV}_{1} \%$.

Methods Of 312 consecutive bronchiectasis patients attending our institution over a 3-year period, 204 patients were suitable for study inclusion. Exclusion criteria consisted of patients with cystic fibrosis or traction bronchiectasis, patients with missing data and patients with absent radiological evidence of bronchiectasis on independent expert thoracic radiologist review. Univariate analyses was performed using Pearson's correlation. Backwards stepwise logistic regression analysis was subsequently performed to determine independent associations of DLCO $\%$ and FEV1\%.

Results DLCO\% strongly correlated with all measured lung function parameters $\mathrm{FEV}_{1} \%$, FVC\%, FEF $25-75 \%$ and $\mathrm{FEV}_{1} / \mathrm{FVC}$ ratio (all $\mathrm{p}<0.001$ ). Negative correlations were noted with age at diagnosis $(\mathrm{p}=0.047)$, body mass index $(\mathrm{p}<0.001)$ and number of comorbidities $(p=0.002)$. Significant symptom associations included SOB $(\mathrm{p}=0.001)$ and fatigue $(\mathrm{p}<0.001)$.
Reduced DLCO\% was associated with higher MRCD scores ( $p<0.001$ ), higher number of hospitalisations on follow up $(p=0.015)$, higher BSI scores $(p<0.001)$ and increased mortality $(p=0.028)$. No correlations were noted with gender, smoking history, aetiology or bacterial colonisation of any form. Reduced DLCO\% was associated with increased number of lobes $(\mathrm{p}=0.004)$, and higher total Reiff and modified Bhalla HRCT scores $(\mathrm{p}=0.001$ and $\mathrm{p}<0.001$ respectively). The modified Bhalla was excluded from stepwise regression as unavailable in most clinical settings. Backwards elimination showed DLCO $\%$ to be significant in predicting BSI $(\mathrm{p}<0.001)$, number of lobes (0.017) and mortality $(\mathrm{p}=0.028)$. Comparatively, backwards stepwise regression of $\mathrm{FEV}_{1} \%$ showed significance in predicting BSI $(p<0.001)$, number of hospitalisations on follow up $(\mathrm{p}=0.008)$, and mortality $(\mathrm{p}=0.024)$. Separate regression models of $\mathrm{DLCO} \%$ and $\mathrm{FEV}_{1} \%$ using modified Bhalla components as cofactors showed DLCO $\%$ to be associated with disease extent $(\mathrm{p}=0.002)$, bronchial wall thickness $(\mathrm{p}<0.001)$, bronchial wall dilatation $(\mathrm{p}<0.001)$ and reduced parenchymal attenuation $(\mathrm{p}=0.015) ; \mathrm{FEV}_{1} \%$ was associated with bronchial wall dilatation only $(\mathrm{p}<0.001)$.

Conclusion DLCO $\%$ predicts radiological disease, disease severity and mortality in bronchiectasis, independently of aetiology, and may identify patients with advanced disease who could benefit from intensive management.

\section{P268 IS BRONCHIECTASIS SEVERITY INFLUENCED BY AETIOLOGY OR CO-MORBID AIRWAYS DISEASE?}

TM Quinn, AT Hill. Royal Infirmary and University of Edinburgh, Edinburgh, UK

\subsection{6/thoraxjnl-2016-209333.411}

Background There is increased interest whether aetiology and co-morbid airways disease influence bronchiectasis disease severity.

Methods We conducted a retrospective study of 400 patients attending a specialist bronchiectasis clinic in NHS Lothian, Edinburgh, UK between May 2013 and September 2014 and using multivariable models we identified independent risk factors that influenced bronchiectasis disease severity using the Bronchiectasis Severity Index. We adjusted for age, sex, smoking history, aetiology and presence of co-morbid airways disease (asthma and COPD).

Results 400 patients were included in this study. The mean age was 66.0 (13.9) years. $253(63.2 \%)$ were female. The majority $(77 \%)$ had idiopathic $(53 \%)$ or post infective bronchiectasis (24\%). Other aetiologies were: allergic bronchopulmonary aspergillosis $8 \%$; immune/auto-immune $6 \%$; interstitial lung disease $3 \%$; ciliary defects $3 \%$; and inflammatory bowel disease $3 \%$. Coexisting airways disease was common but not the predominant diagnosis (36\% had asthma and 19\% COPD).

Independent risk factors for severe bronchiectasis (BSI $\geq 9$ ) were age 70-79 (OR 6.3, $\mathrm{p}=0.003$ ), age 80 and above (OR 7.3, $\mathrm{p}=0.003)$ and smoking (OR 1.02, $\mathrm{p}=0.002)$. It was not influenced by presence of airways disease or aetiology.

Conclusion In conclusion, neither aetiology nor presence of airways disease was independent risk factors for severe bronchiectasis severity. Age was the strongest independent predictor for severe bronchiectasis severity. 